PROCEEDINGS OF THE

AMERICAN MATHEMATICAL SOCIETY

Volume 129, Number 2, Pages 515-524

S 0002-9939(00)05608-2

Article electronically published on August 28, 2000

\title{
ABSOLUTELY CONTINUOUS MEASURES ON NON QUASI-ANALYTIC CURVES WITH INDEPENDENT POWERS
}

\author{
MATS ANDERS OLOFSSON
}

(Communicated by Christopher D. Sogge)

\begin{abstract}
We prove that every non quasi-analytic Carleman class contains functions whose graph supports measures that are absolutely continuous with respect to arc length measure and yet they have independent convolution powers in the measure algebra $M\left(\mathbb{R}^{2}\right)$. The proof relies on conditions which ensure that the canonical map between two Cantor sets can be extended to a function in an arbitrary prescribed non quasi-analytic Carleman class.
\end{abstract}

\section{INTRODUCTION}

Our main technical object with this note is to prove certain sufficient conditions on pairs of Cantor sets $K_{0}$ and $K$ to ensure that the so-called canonical map $\psi: \mathcal{B}_{K_{0}} \rightarrow \mathcal{B}_{K}$ (see Definition 1 in Section [3) can be extended to a function in $C\left\{M_{j}\right\}$, where $C\left\{M_{j}\right\}$ is an arbitrary prescribed non quasi-analytic Carleman class of $C^{\infty}$-functions (see below). In Section 3 our extension result for Cantor set maps is given in three different forms in Theorem 3, Corollary 1 and Theorem 4 The content of those results is that extension is possible if the image Cantor set $K$ is sufficiently small relative to the domain Cantor set $K_{0}$. In some sense these extension results are rather sharp (see Remark 3 in Section 3). Our proofs of those results rely on the methodology used to to prove Whitney's extension theorem (see Stein [10], Chapter VI).

The motivation for this work comes from Björk [1], which was inspired by [2] and [11. Let $A$ be a closed subalgebra (with unit) of the measure algebra $M\left(\mathbb{R}^{n}\right)$. The Fourier transform allows us to consider $\mathbb{R}^{n}$ as a subset of the maximal ideal space of $A$. $A$ is called a Wiener algebra if $\mathbb{R}^{n}$ is dense in the maximal ideal space of $A$; this is known to imply that every measure in $A$ whose Fourier transform is bounded away from zero is invertible in $A$. It is proved in [1] that if $A$ is the closed subalgebra of $M\left(\mathbb{R}^{n}\right)$ generated by all Dirac measures and all measures that are absolutely continuous with respect to Lebesgue surface area measure on some real analytic submanifold of $\mathbb{R}^{n}$, then $A$ is a Wiener algebra. See also Remark 8

Received by the editors April 29, 1999.

2000 Mathematics Subject Classification. Primary 43A10; Secondary 26E10.

Key words and phrases. Measure algebras, Wiener-Pitt phenomenon, independent powers.

The author was supported by the G. S. Magnusson Fund of the Royal Swedish Academy of Sciences. 
in Section 4 In contrast to this we shall prove the following Wiener-Pitt type theorem:

Theorem 1. Let $C\left\{M_{j}\right\}$ be non quasi-analytic. Then there exists a curve

$$
\Gamma=\left\{(x, y) \in \mathbb{R}^{2}: y=f(x)\right\},
$$

where $f \in C\left\{M_{j}\right\}$, and a compactly supported measure $\nu \in M\left(\mathbb{R}^{2}\right)$ absolutely continuous with respect to arc length measure on $\Gamma$ such that $\nu$ has independent powers (Remark 6): Section 4) and is not an element in some Wiener subalgebra $A$ of $M\left(\mathbb{R}^{2}\right)$.

This result sharpens the analogous result in [1] stating the same as Theorem 1 except that the curve $\Gamma$ was only required to be of the class $C^{\infty}$ there. The proof of Theorem 1 is given in Section 4 In the construction used to prove Theorem 1 our extension result Theorem 4 is used in an essential way.

Quasi-analytic classes. Let $\left\{M_{j}\right\}_{j=0}^{\infty}$ be a sequence of positive numbers. We denote by $C\left\{M_{j}\right\}$ the Carleman class consisting of all $C^{\infty}$-functions $f$ on $\mathbb{R}$ satisfying the estimates

$$
\left|f^{(j)}(x)\right| \leq \alpha A^{j} M_{j}, \quad x \in \mathbb{R}, j \geq 0,
$$

for some constants $0<\alpha, A<\infty$. It is known that every such class $C\left\{M_{j}\right\}$ can be defined by means of a logarithmically convex sequence. In fact, $C\left\{M_{j}\right\}=C\left\{\underline{M}_{j}\right\}$, where $\left\{\underline{M}_{j}\right\}_{j=0}^{\infty}$ is the largest logarithmically convex minorant sequence of $\left\{M_{j}\right\}_{j=0}^{\infty}$ (see [6], Chapter V, Section E). Using logarithmic convexity of $\left\{M_{j}\right\}_{j=0}^{\infty}$ one proves that $C\left\{M_{j}\right\}$ is an algebra of functions (see [6], Chapter IV, Section E).

The class $C\left\{M_{j}\right\}$ is said to be quasi-analytic if the zero function $f \equiv 0$ is the only function $f$ in $C\left\{M_{j}\right\}$ satisfying $f^{(j)}(0)=0$ for all $j \geq 0$. The celebrated theorem of Denjoy-Carleman (see [6], Chapter IV, Section B) states that $C\left\{M_{j}\right\}$ is quasi-analytic if and only if

$$
\sum_{j=1}^{\infty} \frac{\underline{M}_{j-1}}{\underline{M}_{j}}=\infty .
$$

A closely related result which will also be referred to as the theorem of DenjoyCarleman is that

$$
\sum_{j=1}^{\infty} \frac{M_{j-1}}{M_{j}}<\infty
$$

implies that $C\left\{M_{j}\right\}$ is non quasi-analytic. In fact, this implication follows from the well-known construction of test functions by means of repeated convolutions of normalized characteristic functions (see [4], Theorem 1.3.2).

\section{WHITNEY EXTENSION OF A FLAT MAP}

The aim with this section is to prove a variant of Whitney's extension theorem (see 10], Chapter VI, Section 2 or [4, Theorem 2.3.6), Theorem 2 below. The novelty of Theorem 2 is that it is formulated in terms of flatness (see assumption (3) below) and Carleman classes. See also Remark 1 below.

Lemma 1. Let $F \subset \mathbb{R}, F \neq \mathbb{R}$, be a non-empty closed set and $C\left\{M_{j}\right\}$ a non quasi-analytic Carleman class. Then there exists a locally finite partition of unity $\left\{\varphi_{k}\right\}_{k=1}^{\infty}$ on $\mathbb{R} \backslash F$ such that: 
1) Every point in $\mathbb{R} \backslash F$ has a neighborhood that intersects at most two of the $\operatorname{supp}\left(\varphi_{k}\right)$ 's.

2) There exists a positive real constant $C$ not depending on $k$ such that:

$$
\operatorname{diam}\left(\operatorname{supp}\left(\varphi_{k}\right)\right) \leq C \operatorname{dist}\left(\operatorname{supp}\left(\varphi_{k}\right), F\right) .
$$

3) There exist constants $0<\alpha, A<\infty$ such that

$$
\left|\varphi_{k}^{(j)}(x)\right| \leq \alpha A^{j} M_{j} \operatorname{dist}(x, F)^{-j}, \quad x \in \mathbb{R}, \quad j=0,1,2, \ldots,
$$

holds for all $k$.

We mention that partitions of unity similar to that in Lemma 1 are constructed in much more generality (arbitrary finite dimensional vector space) by Hörmander in [4] (see Theorem 1.4.10). For the sake of completeness we include a sketch of the proof of Lemma 1

Sketch of the proof of Lemma 1 . Write $\mathbb{R} \backslash F=\bigcup_{k=1}^{\infty} I_{k}$, where the $I_{k}$ 's are closed intervals whose interiors are pairwise disjoint and such that

$$
c_{1} \operatorname{diam}\left(I_{k}\right) \leq \operatorname{dist}\left(I_{k}, F\right) \leq c_{2} \operatorname{diam}\left(I_{k}\right), \quad k=1,2, \ldots,
$$

for some constants $c_{1}$ and $c_{2}$. Let $I_{k}^{*}$ be the interval with the same center as $I_{k}$ but expanded by a factor $1+\varepsilon$ for some $\varepsilon>0$. We now choose $\varepsilon>0$ sufficiently small to ensure that $I_{k}^{*}$ and $I_{j}^{*}$ intersect only if the intervals $I_{k}$ and $I_{j}$ have a common endpoint.

Let $I=[-1,1]$ and $I^{*}=[-(1+\varepsilon), 1+\varepsilon]$. Let $\psi \in C\left\{M_{j}\right\}$ be such that $\psi=1$ on $I, \operatorname{supp}(\psi) \subset I^{*}$ and $0 \leq \psi \leq 1$. (Such a test function $\psi$ can be constructed by convolving a non-negative compactly supported function in $C\left\{M_{j}\right\}$ with integral $=1$ and small support with the characteristic function for an interval.) We now transfer $\psi$ to $I_{k}^{*}$ by defining $\psi_{k}(x)=\psi\left(\left(x-a_{k}\right) / s_{k}\right)$, where $I_{k}=\left[a_{k}-s_{k}, a_{k}+s_{k}\right]$. We now define the $\varphi_{k}$ 's by

$$
\varphi_{1}(x)=\psi_{1}(x), \quad \varphi_{k}(x)=\psi_{k}(x) \prod_{j=1}^{k-1}\left(1-\psi_{j}(x)\right), \quad k \geq 2 .
$$

One checks that $\left\{\varphi_{k}\right\}$ is a partition of unity on $\mathbb{R} \backslash F$. 1) and 2) hold by the construction of the $I_{k}$ and $I_{k}^{*}$ 's. By the construction there are constants $\beta, B$ such that

$$
\left|\psi_{k}^{(j)}(x)\right| \leq \beta B^{j} M_{j} \operatorname{dist}(x, F)^{-j}, \quad x \in \mathbb{R}, \quad j=0,1, \ldots,
$$

holds for all $k \geq 1$. Since locally $\varphi_{k}$ is a product of at most two functions satisfying (2) it follows from the Leibniz formula for the derivative of a product that (1) holds.

Lemma 2. Let $F$ and $\left\{\varphi_{k}\right\}$ be as in Lemma 11. For every $k$, let $p_{k}$ be a point in $F$ closest to $\operatorname{supp}\left(\varphi_{k}\right)$. Then

$$
\left|p_{k}-y\right| \leq(2+C)|x-y|
$$

for all $x \in \operatorname{supp}\left(\varphi_{k}\right)$ and $y \in F$, where $C$ is as in Lemma 1 .

Proof. Choose $x^{*} \in \operatorname{supp}\left(\varphi_{k}\right)$ such that $\left|x^{*}-p_{k}\right|=\operatorname{dist}\left(\operatorname{supp}\left(\varphi_{k}\right), F\right)$. By part 2$)$ of Lemma 1 we have

$$
\left|p_{k}-y\right| \leq\left|p_{k}-x^{*}\right|+\left|x^{*}-x\right|+|x-y| \leq(2+C)|x-y| .
$$


Theorem 2. Let $C\left\{M_{j}\right\}$ be non quasi-analytic. Let $F \subset \mathbb{R}, F \neq \mathbb{R}$, be a nonempty closed set and $\psi$ a continuous function on $F$ such that

$$
|\psi(x)-\psi(y)| \leq \Lambda_{j}|x-y|^{j}, \quad x, y \in F, \quad j=1,2, \ldots,
$$

for some constants $0<\Lambda_{j}<\infty$.

Then $\psi$ has an extension to a $C^{\infty}$-function $\Psi$ on $\mathbb{R}$ satisfying the estimates

$$
\left|\Psi^{(j)}(x)\right| \leq 2 \alpha A^{j}(2+C)^{j} \Lambda_{j} M_{j}, \quad x \in \mathbb{R}, \quad j=1,2, \ldots,
$$

where $\alpha, A$ and $C$ are as in Lemma 1. If $\psi$ is bounded, then $\Psi \in C\left\{\Lambda_{j} M_{j}\right\}$.

Proof. Choose $\left\{\varphi_{k}\right\}_{k=1}^{\infty}$ and $\left\{p_{k}\right\}_{k=1}^{\infty}$ as in the preceding lemmas. The extension $\Psi$ of $\psi$ is now defined by

$$
\Psi(x)= \begin{cases}\sum_{k=1}^{\infty} \psi\left(p_{k}\right) \varphi_{k}(x), & x \notin F \\ \psi(x), & x \in F\end{cases}
$$

It is clear that $\Psi$ is $C^{\infty}$ in $\mathbb{R} \backslash F$ and that $\Psi^{(j)}$ exists and is $=0$ in the interior of $F$ for all $j \geq 1$. For proving that $\Psi$ is $C^{\infty}$ it suffices to check that every $\Psi^{(j)}$ extends continuously to $\mathbb{R}$.

The case $j=0$. It suffices to prove that $F \not \supset x \rightarrow y \in F$ implies $\Psi(x) \rightarrow \psi(y)$. We have that

$$
\Psi(x)-\psi(y)=\sum_{k}\left(\psi\left(p_{k}\right)-\psi(y)\right) \varphi_{k}(x) .
$$

By (3) and Lemma 2,

$$
|\Psi(x)-\psi(y)| \leq \sum_{k} \Lambda_{1}\left|p_{k}-y\right| \varphi_{k}(x) \leq(2+C) \Lambda_{1}|x-y| \rightarrow 0
$$

as $x \rightarrow y$.

The case $j>0$. Assume $x \notin F$. Choose $y \in F$ as close to $x$ as possible. Since $\sum_{k} \varphi_{k}^{(j)}(x)=0$ we have that

$$
\Psi^{(j)}(x)=\sum_{k} \psi\left(p_{k}\right) \varphi_{k}^{(j)}(x)=\sum_{k}\left(\psi\left(p_{k}\right)-\psi(y)\right) \varphi_{k}^{(j)}(x)
$$

By (3) and Lemma 2,

$$
\left|\Psi^{(j)}(x)\right| \leq \sum_{k} \Lambda_{j+1}\left|p_{k}-y\right|^{j+1}\left|\varphi_{k}^{(j)}(x)\right| \leq \Lambda_{j+1}(2+C)^{j+1}|x-y|^{j+1} \sum_{k}\left|\varphi_{k}^{(j)}(x)\right| .
$$

By (11) and part 1) of Lemma 1] we can estimate $\sum_{k}\left|\varphi_{k}^{(j)}(x)\right|$ by $2 \alpha A^{j} M_{j}|x-y|^{-j}$. We conclude that

$$
\left|\Psi^{(j)}(x)\right| \leq 2 \alpha A^{j}(2+C)^{j+1} \Lambda_{j+1} M_{j}|x-y| \rightarrow 0
$$

as $x \rightarrow F$.

We now prove (4). Assume $x \notin F$. Choose $y \in F$ as close to $x$ as possible. By (5) we have

$$
\left|\Psi^{(j)}(x)\right| \leq \sum_{k} \Lambda_{j}\left|p_{k}-y\right|^{j}\left|\varphi_{k}^{(j)}(x)\right| \leq \Lambda_{j}(2+C)^{j}|x-y|^{j} \sum_{k}\left|\varphi_{k}^{(j)}(x)\right| .
$$

Estimating $\sum_{k}\left|\varphi_{k}^{(j)}(x)\right|$ by $2 \alpha A^{j} M_{j}|x-y|^{-j}$ we get (4). 
Remark 1. The case of interest in Theorem 2 is when the set $F$ is highly irregular. For instance, if $F=[0,1]$, then the assumption (3) for $j=2$ implies that $\psi$ is constant. In the proof of Theorem 3 in Section 3 we apply Theorem 2 to the case when $F$ is a Cantor set and $\psi$ is a so-called canonical map (Definition [1: Section 3).

\section{Extension of MAPS Between CANTOR Sets}

A class of Cantor sets on $[0,1]$. For every $n \geq 1$ let there be given $2^{n-1}$ open intervals

such that

$$
\Delta_{j}^{(n)}=\left(\alpha_{j}^{(n)}, \beta_{j}^{(n)}\right), \quad j=1, \ldots, 2^{n-1},
$$

$$
0<\alpha_{1}^{(n)}<\beta_{1}^{(n)}<\alpha_{2}^{(n)}<\beta_{2}^{(n)}<\cdots<\alpha_{2^{n-1}}^{(n)}<\beta_{2^{n-1}}^{(n)}<1 .
$$

Write $S^{(0)}=[0,1]=S_{1}^{(0)}$ and set

$$
S^{(n)}=S^{(n-1)} \backslash \bigcup_{j=1}^{2^{n-1}} \Delta_{j}^{(n)}=\bigcup_{j=1}^{2^{n}} S_{j}^{(n)}, \quad n \geq 1,
$$

where the $S_{j}^{(n)}, 1 \leq j \leq 2^{n}$, are pairwise disjoint closed intervals. We demand further in the construction that the intervals $\Delta_{j}^{(n)}$ are such that

$$
\Delta_{j}^{(n+1)} \subset S_{j}^{(n)}, \quad 1 \leq j \leq 2^{n}, n \geq 1 .
$$

The corresponding Cantor set is now defined by

$$
K=\bigcap_{n=0}^{\infty} S^{(n)}=[0,1] \backslash \bigcup_{n=1}^{\infty} \bigcup_{j=1}^{2^{n-1}} \Delta_{j}^{(n)} .
$$

We define

$$
\begin{gathered}
\lambda_{K}(n)=\max _{1 \leq j \leq 2^{n}}\left|S_{j}^{(n)}\right| \quad(n \geq 0), \\
\delta_{K}(n)=\min _{1 \leq j \leq 2^{n-1}}\left|\Delta_{j}^{(n)}\right| \quad(n \geq 1)
\end{gathered}
$$

and

$$
\mathcal{B}_{K}=\left\{0,1, \alpha_{j}^{(n)}, \beta_{j}^{(n)}: 1 \leq j \leq 2^{n-1}, n \geq 1\right\} .
$$

We call $K$ an admissible Cantor set if $K$ is constructed as above and $\lambda_{K}(n) \rightarrow 0$ as $n \rightarrow \infty$.

Remark 2. Note that $\mathcal{B}_{K}$ is dense in $K$ if $K$ is an admissible Cantor set. Let us also notice that there exist admissible Cantor sets with positive Lebesgue measure. For example when every $\Delta_{j}^{(n)}, 1 \leq j \leq 2^{n-1}$, has length $1 / 4^{n}$ and the $S_{j}^{(n)}, 1 \leq j \leq 2^{n}$, are of equal length, then the above construction gives an admissible Cantor set of measure $1 / 2$.

For easy reference we record the following lemma whose proof is trivial.

Lemma 3. Let $K$ be an admissible Cantor set on $[0,1]$. Let $x, y \in K, x \neq y$, and denote by $n(x, y)$ the largest integer $n \geq 0$ such that both $x \in S_{j}^{(n)}$ and $y \in S_{j}^{(n)}$ for some $j$. Then,

$$
\delta_{K}(n(x, y)+1) \leq|x-y| \leq \lambda_{K}(n(x, y)) .
$$


Definition 1. Let $K_{0}$ and $K$ be admissible Cantor sets on [0,1], where $K$ is constructed as above and $K_{0}$ is obtained at step $n \geq 1$ by removing the intervals

$$
\left(a_{j}^{(n)}, b_{j}^{(n)}\right), \quad j=1, \ldots, 2^{n-1} .
$$

The canonical map $\psi: \mathcal{B}_{K_{0}} \rightarrow \mathcal{B}_{K}$ is defined by $\psi(0)=0, \psi(1)=1, \psi\left(a_{j}^{(n)}\right)=\alpha_{j}^{(n)}$, $\psi\left(b_{j}^{(n)}\right)=\beta_{j}^{(n)}$.

Note that $\psi$ is strictly increasing and thereby preserves the construction.

Proposition 1. Let $K_{0}$ and $K$ be admissible Cantor sets on $[0,1]$ and let $\psi$ : $\mathcal{B}_{K_{0}} \rightarrow \mathcal{B}_{K}$ be the corresponding canonical map (Definition 11). Then the following estimate holds:

$$
|\psi(x)-\psi(y)| \leq \Lambda_{j}|x-y|^{j}, \quad x, y \in \mathcal{B}_{K_{0}}, \quad j=0,1,2, \ldots,
$$

where

$$
\Lambda_{j}=\sup _{n \geq 0} \frac{\lambda_{K}(n)}{\delta_{K_{0}}(n+1)^{j}} .
$$

Proof. Since $\psi$ is strictly increasing, $n(\psi(x), \psi(y))=n(x, y)$, where $n(\cdot, \cdot)$ is as in Lemma 3. For $x, y \in \mathcal{B}_{K_{0}}, x \neq y$, we have

$$
\begin{gathered}
|\psi(x)-\psi(y)| \leq \lambda_{K}(n(\psi(x), \psi(y)))=\frac{\lambda_{K}(n(x, y))}{|x-y|^{j}}|x-y|^{j} \\
\leq \frac{\lambda_{K}(n(x, y))}{\delta_{K_{0}}(n(x, y)+1)^{j}}|x-y|^{j} \leq \Lambda_{j}|x-y|^{j} .
\end{gathered}
$$

We are mainly interested in the case when $\Lambda_{j}<\infty$ for all $j$. Note however that $\psi$ extends uniquely to a Lipschitz continuous function $\psi: K_{0} \rightarrow K$ if $\Lambda_{1}<\infty$.

Remark 3. Let the notation be as in Proposition 1 and assume that $\Lambda_{j}<\infty$ for every $j$. If $\Psi$ is a $C^{\infty}$-extension of $\psi$, then $\Psi^{(j)} \equiv 0$ on $K_{0}$ for every $j \geq 1$. In particular, such an extension $\Psi$ (if it exists) cannot be in some quasi-analytic class. In Theorem 4 below we show that if $\lambda_{K}(n) \rightarrow 0$ sufficiently fast, then there exists an extension $\Psi \in C\left\{M_{j}\right\}$ of $\psi$, where $C\left\{M_{j}\right\}$ is an arbitrary prescribed non quasi-analytic Carleman class.

Also, since $\Psi^{(j)}(0)=0$ for all $j \geq 0, \Psi$ can be glued together with any $C^{\infty}$ function, all of whose derivatives vanish at 0 to form a $C^{\infty}$-extension of $\psi$.

Applying Theorem 2 to the canonical map between two Cantor sets we obtain:

Theorem 3. Let $C\left\{M_{j}\right\}$ be non quasi-analytic and let $\left\{\Lambda_{j}\right\}_{j=0}^{\infty}$ be a sequence of positive real numbers with $\lim _{j \rightarrow \infty} \Lambda_{j}^{1 / j}=\infty$. Let $K_{0}$ and $K$ be admissible Cantor sets on $[0,1]$ and $\psi: \mathcal{B}_{K_{0}} \rightarrow \mathcal{B}_{K}$ be the corresponding canonical map (Definition $[1$ ).

If the set $K$ is constructed in such a way that

$$
\lambda_{K}(n) \leq \min _{j \geq 0} \delta_{K_{0}}(n+1)^{j} \Lambda_{j}
$$

for all $n$ sufficiently large, then $\psi$ has an extension to a function $\Psi$ in $C\left\{\Lambda_{j} M_{j}\right\}$. 
Proof. By (8) and Proposition 1 we can uniquely extend $\psi$ to a continuous function $\psi: K_{0} \rightarrow K$ such that for some $C>0$,

$$
|\psi(x)-\psi(y)| \leq C^{j+1} \Lambda_{j}|x-y|^{j}, \quad j \geq 0,
$$

for all $x, y \in K_{0}$. By Theorem [2] has an extension to a function $\Psi$ in the class $C\left\{C^{j+1} \Lambda_{j} M_{j}\right\}=C\left\{\Lambda_{j} M_{j}\right\}$.

Remark 4. The assumption on $\left\{\Lambda_{j}\right\}_{j=0}^{\infty}$ that $\lim _{j \rightarrow \infty} \Lambda_{j}^{1 / j}=\infty$ is needed to guarantee that the minima in (8) are attained.

Corollary 1. Let $C\left\{M_{j}\right\}$ be non quasi-analytic. Let $K_{0}, K$ and $\psi$ be as in Theorem 3. Let

$$
\Lambda_{j}=\sup _{n \geq 0} \frac{\lambda_{K}(n)}{\delta_{K_{0}}(n+1)^{j}} .
$$

If $C\left\{M_{j} / \Lambda_{j}\right\}$ is non quasi-analytic, then $\psi$ has an extension to a function $\Psi$ in $C\left\{M_{j}\right\}$.

Proof. Let $L_{j}=M_{j} / \Lambda_{j}$. By our choice of $\Lambda_{j}$,

$$
\lambda_{K}(n) \leq \inf _{j \geq 0} \delta_{K_{0}}(n+1)^{j} \Lambda_{j}
$$

By Theorem 3 we see that $\psi$ extends to a function in $C\left\{\Lambda_{j} L_{j}\right\}=C\left\{M_{j}\right\}$.

The assumption in Corollary 1 that $C\left\{M_{j} / \Lambda_{j}\right\}$ is non quasi-analytic is, by the Denjoy-Carleman theorem, satisfied if

$$
\sum_{j=1}^{\infty} \frac{\Lambda_{j}}{\Lambda_{j-1}} \frac{M_{j-1}}{M_{j}}<\infty .
$$

This condition is fulfilled if $\lambda_{K}(n) \rightarrow 0$ sufficiently fast. We state this explicitly in the next theorem.

Theorem 4. Let $C\left\{M_{j}\right\}$ be non quasi-analytic and let $K_{0}$ be an admissible Cantor set on $[0,1]$.

If $K$ is an admissible Cantor set on $[0,1]$ such that $\lambda_{K}(n) \rightarrow 0$ sufficiently fast (the rate of convergence depends only on $\left\{\delta_{K_{0}}(n)\right\}_{n=1}^{\infty}$ and $\left\{M_{j}\right\}_{j=0}^{\infty}$ ), then the canonical map $\psi: \mathcal{B}_{K_{0}} \rightarrow \mathcal{B}_{K}$ can be extended to a function $\Psi$ in $C\left\{M_{j}\right\}$.

Proof. We may assume that $\left\{M_{j}\right\}_{j=0}^{\infty}$ is logarithmically convex. Since, by the theorem of Denjoy-Carleman, $\sum M_{j-1} / M_{j}<\infty$, we can choose $0<\omega_{j} \rightarrow \infty$ such that

$$
\sum_{j=1}^{\infty} \omega_{j} \frac{M_{j-1}}{M_{j}}<\infty
$$

Set $\Lambda_{0}=1$ and

$$
\Lambda_{j}=\prod_{l=1}^{j} \omega_{l}, \quad j \geq 1 .
$$

By the theorem of Denjoy-Carleman (the non log-convex case, see the introductory remark on quasi-analytic classes), (9) implies that the class $C\left\{M_{j} / \Lambda_{j}\right\}$ is non quasianalytic. 
If $K$ is such that

$$
\lambda_{K}(n) \leq \min _{j \geq 0} \delta_{K_{0}}(n+1)^{j} \Lambda_{j}, \quad n \text { large }
$$

then by Theorem 3, $\psi$ extends to a function in $C\left\{\Lambda_{j} M_{j} / \Lambda_{j}\right\}=C\left\{M_{j}\right\}$.

\section{Application to Wiener algebras}

In this section we give the proof of Theorem 1 in the introduction. First we state some preliminary results.

Lemma 4. There exists a Cantor set $K$ on $[0,1]$ such that $K \backslash\{0\}$ is linearly independent over $\mathbb{Q}$ and $\lambda_{K}(n) \rightarrow 0$ faster than any prescribed quantity.

Proof. By translating and dilating, this lemma is an immediate consequence of the construction of a linearly independent Cantor set (see [3], pages 187-189 or [5], pages 20-21).

Lemma 5 (Theorem 5.3.2 in [8]). Let $G$ be a non-discrete locally compact abelian group and $E$ an independent (see Remark 5) compact subset of $G$. If $\mu \in M(G)$ is a continuous measure concentrated on $E \cup(-E)$, then the measures $\delta_{0}, \mu, \mu^{* 2}, \mu^{* 3}, \ldots$ are mutually singular.

Remark 5. A set $E \subset G$ is said to be independent if for every choice of distinct points $x_{1}, \ldots, x_{k}$ in $E$ and $n_{1}, \ldots, n_{k} \in \mathbb{Z}, n_{1} x_{1}+\cdots+n_{k} x_{k}=0$ implies $n_{1} x_{1}=$ $\cdots=n_{k} x_{k}=0$.

Remark 6 . Recall that $\mu \in M(G)$ is said to have independent powers if the measures $\delta_{0}, \mu, \mu^{* 2}, \ldots$ are mutually singular.

Below we use the notation $\sigma_{A}(x)$ for the spectrum of the element $x$ in the Banach algebra $A$.

Lemma 6. Let $A$ be a closed subalgebra of a Banach algebra B. Let $x \in A$. Then the boundary of $\sigma_{A}(x)$ is contained in $\sigma_{B}(x)$.

Proof. This result is well known and can be found as part of Theorem 10.18 in [9].

Proof of Theorem 1. Let $C\left\{M_{j}\right\}$ be non quasi-analytic. Let $K_{0}$ be an admissible Cantor set on $[0,1]$ with positive Lebesgue measure (see Remark 2] in Section 3). By Lemma 4 we can find an admissible Cantor set $K$ on $[0,1]$ such that $K \backslash\{0\}$ is linearly independent over $\mathbb{Q}$ and $\lambda_{K}(n) \rightarrow 0$ sufficiently fast in the sense of Theorem 4. By Theorem [ the canonical map $\psi: \mathcal{B}_{K_{0}} \rightarrow \mathcal{B}_{K}$ (Definition 1 Section (3) has an extension to a function $\Psi$ in the class $C\left\{M_{j}\right\}$. Let $P=K_{0} \times K$ and define

$$
f(x)=\left\{\begin{aligned}
\Psi(x) & \text { if } \quad x \geq 0 \\
-\Psi(-x) & \text { if } \quad x \leq 0
\end{aligned}\right.
$$

Then, by Remark 3 is a function in $C\left\{M_{j}\right\}$. Let

$$
\Gamma=\left\{(x, y) \in \mathbb{R}^{2}: y=f(x), x \in \mathbb{R}\right\}
$$

be the graph of $f$. Let $\sigma$ denote the arc length measure on $\Gamma$. Define the measure $\nu$ by

$$
\nu(E)=\sigma(E \cap(P \cup(-P)))
$$


for every Borel set $E$ in $\mathbb{R}^{2}$. It is obvious that $\nu$ is absolutely continuous with respect to $\sigma$. The proof is now completed by the following theorem:

Theorem 5. In the above situation, $\nu$ has independent powers (Remark 6: Section (4) and is not an element in some Wiener subalgebra $A$ of $M\left(\mathbb{R}^{2}\right)$.

Proof. By Lemma 5 with $G=\mathbb{R}^{2}$ and $E=P \cap \Gamma=\left\{(x, f(x)): x \in K_{0}\right\}, \nu$ has independent powers. Using Remark 3 a computation shows that the action of $\nu$ on test functions is given by

$$
\int \varphi d \nu=\int_{K_{0} \cup\left(-K_{0}\right)} \varphi(x, f(x)) d x .
$$

Since $K_{0}$ has positive Lebesgue measure, $\nu \neq 0$. Assume for reaching a contradiction that $\nu \in A$ for some Wiener algebra $A$ in $M\left(\mathbb{R}^{2}\right)$. Since $f$ is odd it follows from (10) that the Fourier transform of $\nu$ is real-valued. Since $A$ is Wiener, $\sigma_{A}(\nu) \subset \mathbb{R}$.

Let $A_{0}$ be the closed subalgebra of $M\left(\mathbb{R}^{2}\right)$ generated by $\nu$. Since $\nu$ has independent powers, $A_{0}$ consists of all measures of the form

$$
\mu=\sum_{j=0}^{\infty} a_{j} \nu^{* j},
$$

with

$$
\|\mu\|=\sum_{j=0}^{\infty}\left|a_{j}\right|\|\nu\|^{j}<\infty .
$$

It is well known that the multiplicative linear functionals on such an algebra are of the form

$$
\mu \mapsto \sum_{j=0}^{\infty} a_{j} \lambda^{j}
$$

where $\lambda$ is a complex number such that $|\lambda| \leq\|\nu\|$ and $\mu$ is given by (11). In particular,

$$
\sigma_{A_{0}}(\nu)=\{\lambda \in \mathbb{C}:|\lambda| \leq\|\nu\|\} .
$$

By Lemma 6 this implies that

$$
\{\lambda \in \mathbb{C}:|\lambda|=\|\nu\|\} \subset \sigma_{A}(\nu),
$$

which contradicts $\sigma_{A}(\nu) \subset \mathbb{R}$.

Remark 7. By (10), $\nu$ is the push-forward measure $F_{*}\left(d x \mid K_{0}\right)$, where $F: x \mapsto$ $(x, f(x))$.

Remark 8. The strongest result in [1] states that if $A$ is the closed subalgebra of $M\left(\mathbb{R}^{n}\right)$ generated by all discrete measures and all measures that are absolutely continuous with respect to Lebesgue surface area measure on some $C^{1}$-submanifold of $\mathbb{R}^{n}$ in the so-called generic position, then $A$ is a Wiener algebra. A consequence of this result is that if $B$ is the closed subalgebra of $M\left(\mathbb{R}^{2}\right)$ generated by all discrete measures, $L^{1}\left(\mathbb{R}^{2}\right)$ and all measures that are absolutely continuous with respect to arc length measure on some quasi-analytic curve $\gamma$ in $\mathbb{R}^{2}$, then $B$ is a Wiener algebra. Here the assertion that $\gamma$ is a quasi-analytic curve is to be understood in the sense that the components of $\gamma$, considered as functions on the parameter interval, are both members in some linear quasi-analytic class of $C^{\infty}$-functions. An exposition of the above results by Björk and some related matters will appear in [7]. 


\section{ACKNOWLEDGEMENT}

The author is grateful to Jan-Erik Björk and Jan Boman for their valuable suggestions regarding this work.

\section{REFERENCES}

1. J-E. Björk, Wiener Subalgebras of $M\left(\mathbb{R}^{n}\right)$ Generated by Smooth Measures Carried by Smooth Submanifolds of $\mathbb{R}^{n}$, preprint, Stockholm University, 1979.

2. J. Boman, Equivalence of Generalized Moduli of Continuity, Ark. Mat. 18 (1980) 73-100. MR 82m:42012

3. I. Gelfand, D. Raikov and G. Shilov, Commutative Normed Rings, Chelsea Publishing Company, 1964. MR 34:4940

4. L. Hörmander, The Analysis of Linear Partial Differential Operators I, second edition, Springer, 1990.

5. J-P Kahane and R. Salem, Ensembles parfaits et séries trigonométriques, Hermann, 1963. MR 28:3279

6. P. Koosis, The Logarithmic Integral I, Cambridge University Press, 1988. MR 99j:30001

7. A. Olofsson, Nilpotent Measures and Wiener Subalgebras of $M\left(\mathbb{R}^{n}\right)$, paper IV in Topics in Real and Complex Analysis, doctoral thesis, Stockholm University, 2000.

8. W. Rudin, Fourier Analysis on Groups, John Wiley \& Sons, 1990. MR 91b:43002

9. W. Rudin, Functional Analysis, McGraw-Hill, 1973. MR 51:1315

10. E. M. Stein, Singular Integrals and Differentiability Properties of Functions, Princeton University Press, 1970

11. N. Varopoulos, Studies in Harmonic Analysis, Proc. Camb. Phil. Soc. 60 (1964) 465-516. MR 29:1284

Department of Mathematics, Stockholm University, SE-106 91 Stockholm, Sweden

E-mail address: anderso@matematik.su.se 